\title{
Anthropologie et développement en Belgique
}

\section{Michael Singleton}

\section{(2) OpenEdition}

Journals

Édition électronique

URL : http://journals.openedition.org/apad/305

DOI : 10.4000/apad.305

ISSN : 1950-6929

\section{Éditeur}

LIT Verlag

Édition imprimée

Date de publication : 15 mars 1991

\section{Référence électronique}

Michael Singleton, «Anthropologie et développement en Belgique », Bulletin de l'APAD [En ligne], 1।

1991, mis en ligne le 23 juin 2006, consulté le 08 septembre 2020. URL : http://

journals.openedition.org/apad/305; DOI : https://doi.org/10.4000/apad.305

Ce document a été généré automatiquement le 8 septembre 2020.

Bulletin de l'APAD 


\title{
Anthropologie et développement en Belgique
}

\author{
Michael Singleton
}

Préambule pour bien situer celui qui parle

1 Pour passer le temps au-dessus d'un Sahara harmattanisé, je feuilletais mon Sabena "in flight magazine". En dernière page utile parmi les questions d'un petit quizz : "Quel est le pays qui exporte le plus au monde par rapport à sa population et son PNB ?" Vu le contexte, pas besoin d'être grand clerc pour savoir que c'était ni le Japon, ni l'Allemagne, mais la "petite" Belgique. "Exporter ou ne pas être" - voilà la question vitale à quoi doivent répondre des pays de taille modeste coincés par les lois du marché capitaliste qui les gouvernent.

2 C'est peut-être ce même mécanisme qui fait qu'il y a plus d'anthropologues belges à l'étranger que chez eux. Et ce ne sont pas les moindres: un Vansina en Amérique, Heusch en France pour ne citer qu'eux (à moins qu'ils ne se soient expatriés pour échapper aux séquelles des querelles ethnolinguistique de rigueur dans leur réserve indigène ?). C'est sans doute en partie cette fuite de ses cerveaux qui a obligé la Belgique à importer des anthropologues - entre autres l'anglo-belge responsable de cette note. D'un certain point de vue - celui, d'ailleurs, largement partagé par la plupart de mes collègues belgo-belges - peu importe, à la limite, dans une Europe qui se veut régionale plutôt que nationaliste, toutes ces questions de provenance géo-politique et d'appartenance ethnique, pourvu qu'il y ait des anthropologues un peu partout à la fois.

3 Communication spontanée et instantanée même lors des journées de lancement de l'APAD, ce bref survol de ce qui se fait en matière d"anthropologie et développement" en Belgique reste impressionniste et subjectif. D'abord parce que je n'ai pas reçu de mandat pour faire un état exhaustif des lieux. Ensuite parce que de retour en Belgique et en réfléchissant à tête plus reposée aux personnes qui se préoccupent du développement et aux organisations qui s'en occupent, je suis sidéré par leur grande quantité et : leur haute qualité. D'où la nécessité pour éviter tout débordement diluvien de ne faire état que des lieux consacrés ex professo (surtout du côté francophone) à 
l'anthropologie du développement - africain en plus. Les sympathisants du dehors pourraient faire l'objet d'un bilan ultérieur !

Au niveau national

4 Le Musée de Tervuren (banlieue sud de Bruxelles), qui a son origine dans une exposition concoctée par Léopold à la fin du siècle passé pour montrer ce qui se faisait dans son Congo, a toujours été plus qu'un simple dépotoir et continu d'être branché directement sur le développement. avec un faible pour l'Afrique Centrale.

5 Dans la capitale même: la Bibliothèque Africaine, en plus d'une collection incontournable pour l'histoire de l'Afrique Centrale, constitue un outil indispensable pour suivre l'évolution de l'Afrique contemporaine. Le CEDAF (Centre d'Études et de Documentation Africaine) publie des ouvrages sur des questions de développement ayant une affinité certaine avec l'approche anthropologique. Tandis que l'Académie Royale des Sciences d'Outremer fait preuve d'un intérêt plus qu' "académique" pour l'Afrique - ayant organisé récemment par exemple un colloque sur le Cameroun et sponsorisé des enquêtes sur la situation alimentaire au Zaïre. Enfin l'AGCD (l'Agence Générale pour la Coopération au Développement du ministère de la coopération), en plus de son appui a des ONG de formation et d'information para-universitaires, organise des stages de préparation pour des coopérants qui sont confiés pour l'essentiel à une équipe fortement anthropologisée (il était question a un certain moment de regrouper tout cela sous le chapeau d'un Institut Africain, mais je ne sais pas si la question a connu de réponse en ce sens.)

$\mathrm{Au}$ niveau universitaire

6 Coté francophone : à l'Université Catholique de Louvain (UCL), dans le domaine des sciences humaines : à côté d'une unité proprement anthropologique, il y a un Institut des Pays en voie de Développement ayant un volet africanistique assez conséquent ; un diplôme en Études Africaines ; un programme axé autour des projets de développement (surtout africains) et organisé par la FOPES-sud; le CIDEP (Centre International de Recherche et de Formation en Population et Développement) en association avec les Nations-Unies (FNUAP); le département de Démographie qui a toujours eu non seulement un public africain, mais un intérêt certain pour le développement et les recherches qualitatives (il est à noter que pas mal de ces lieux vont se retrouver d'une façon ou d'une autre dans un nouveau département qui démarre en l'année académique 91-92).

7 Du côté des sciences naturelles et/ou appliquées: les médecins et les agronomes continuent à se pencher sur l'Afrique, mais ce sont peut-être les architectes et les aménagistes groupés autour de la cellule "Habitat et Développement" qui ont le plus affaire aux anthropologues.

8 "Last but not least" de (jeunes) chercheurs se sont groupés dans une association APPROCHE et si on voulait être complet il faudrait faire allusion aux associations d'étudiants africains et belges centrés sur un "esprit anthropologique" en rapport avec le développement de l'Afrique.

"Charité bien ordonnée commençant chez soi", j'ai détaillé d'abord ce qui vient à mon esprit en matière d'Anthropologie et Développement en pensant à mon université, mais plus parce que cela illustre ce qui se fait en gros sur les autres campus belges que par provincialisme publicitaire. Car on trouve - parfois en plus grand, parfois en plus petit les même créneaux sur la plupart des autres campus belges: l'Université Libre de Bruxelles possède une unité d'anthropologie qui a "inspiré" en partie des associations 
de jeunes chercheurs : APRAD (Association Pluridisciplinaire pour la Recherche-Action en matière de Développement) et CISCOD (Cercle d'Information et de Réflexion sur le Service Civil et la Coopération au Développement) ; l'ULB a aussi une a.s.b.l. (la CODULB - Coopération au Développement); à son tour l'Université de Liège s'est dotée d'une a.s.b.l. pour le développement (l'ACDST) et gère des réseaux du genre R.E.N.A. (Réseau pour l'Éducation Nutritionnelle en Afrique); rattachée aux Facultés Universitaires de Namur, une ONG, la FUCID (Fondation Universitaire pour la Coopération Internationale pour le Développement) a réalisé des recherches-actions en Afrique auxquelles des anthropologues ont été associés; dans le cadre du réseau international PRELUDE (Programme de Recherche et de Liaison Universitaire pour le Développement coordonné à partir de-Namur) j'ai eu l'occasion de me familiariser avec ce qui se faisait à l'université de Liège, aux Facultés d'Agronomie de Gembloux, à la Fondation Universitaire Luxembourgeoise d'Arlon et à Mons dans des domaines qui sont au cœur du projet $A P A D$, mais les lister ici nous amènerait trop loin.

Côté néerlandophone : à ma connaissance la Katholieke Universiteit Leuven (KUL) est la seule à avoir un département d'anthropologie en bonne et due forme (qui a un penchant pour l'anthropologie non seulement africaine mais médicale); les agronomes et les architectes de la KUL, pour ne parler que d'eux, se sont engagés dans le développement africain; ailleurs en Flandres, à Gand, il y a un Institut d'Erémologie qui, s'occupant des régions désertiques ou en voie de désertification, ne peut guère être indifférent aux pays sahéliens et aux facteurs anthropologiques ; les démographes de la Vrij Universiteit Brussel (VUB) - pour ne parler de nouveau que d'eux - s'occupent particulièrement des problèmes africains; enfin, à Anvers, en plus des spécialistes du développement africain au Rijksuniversiteit (qui comme la plupart d'autres campus belges connaît une population estudiantine africaine appréciable), il ne faut pas oublier l'Institut de Médecine Tropicale avec des unités comme celle de l'URESP (Unité de Recherche en Santé Publique) faisant preuve d'une sensibilité certaine à la dimension dite "culturelle".

Ailleurs et autrement

11 Il fallait bien partir de quelque part et nous sommes parti des centres d'où, au mieux, l'anthropologie professionnelle et l'africanistique disciplinée rayonnent - vers les sphères périphériques de la politique, du privé et du philanthropique. Mais attention à l'illusion optique et surtout à l'illusion d'optiques. On a beau être au centre de son monde, il n'est pas dit que son monde soit le centre du monde le plus pertinent ou performant. A supposer qu'il soit plus qu'une filière pour sortir les anthropologues de leur impasse et une façon pour les africanistes de résoudre leur crise d'identité, l'offre de l'APAD pourrait rencontrer une demande explicite d'autres acteurs sur la scène africaine. On pourrait même imaginer que l'APAD vient, à point nommé, cristalliser les aspirations d'autres personnages à mieux figurer dans la même pièce. Il est permis de rêver... en dépit de la réalité qui veut que chacun ait son Afrique, que l'Afrique "réelle" fait les frais des images qu'on se donne d'elle. Et il n'est pas dit que l'Afrique offerte par le politicien (politicard ou pas), le privé (profiteur ou pas), le philanthrope (partisan ou pas), l'industriel, convienne à moins d'Africains que l'Afrique de l'anthropologue ou de l'africaniste!

Ceux qui se penchent sur l'Afrique et qui pensent à son "développement" se divisent en au moins trois parties : d'abord, il y a ceux susceptibles de souscrire à la vision et aux valeurs de l'APAD, ensuite il y a ceux qui pourraient être tentés par ce genre de 
philosophe et de pratique, et, enfin, il y a tout un monde porteur de programmes et de projets peu apadiens. Ici je n'ai inventorié qu'un échantillon des premiers, je soupçonne l'existence des seconds, mais je crains qu'ils représentent plus un glaçon en débâcle que la partie émergée d'un immense iceberg. 